Please do not remove this page

RMIT

UNIVERSITY

\title{
Inertia effect on buckling-induced auxetic metamaterials
}

Shen, Jianhu; Zhou, Shiwei; Huang, Xiaodong; Ruan, Dong; Xie, Yimin

https://researchrepository.rmit.edu.au/esploro/outputs/9921862765801341/filesAndLinks?institution=61RMIT_INST\&index=null

Shen, J., Zhou, S., Huang, X., Ruan, D., \& Xie, Y. (2015). Inertia effect on buckling-induced auxetic metamaterials. International Journal of Protective Structures, 6(2), 311-322.

https://doi.org/10.1260/2041-4196.6.2.311

Document Version: Accepted Manuscript

Published Version: https://doi.org/10.1260/2041-4196.6.2.311

Repository homepage: https://researchrepository.rmit.edu.au

Downloaded On 2023/04/26 23:59:47 +1000

Please do not remove this page 
Thank you for downloading this document from the RMIT Research Repository.

The RMIT Research Repository is an open access database showcasing the research outputs of RMIT University researchers.

RMIT Research Repository: http://researchbank.rmit.edu.au/

\author{
Citation: \\ Shen, J, Zhou, S, Huang, X, Ruan, D and Xie, Y 2015, 'Inertia effect on \\ buckling-induced auxetic metamaterials', International Journal of Protective \\ Structures, vol. 6, no. 2, pp. 311-322.
}

See this record in the RMIT Research Repository at:

https://researchbank.rmit.edu.au/view/rmit:32185

Version: Accepted Manuscript

Copyright Statement:

(c) N/A

Link to Published Version:

http://dx.doi.org/10.1260/2041-4196.6.2.311 


\title{
Inertia Effect on Buckling-induced Auxetic Metamaterials
}

\author{
Jianhu Shen*†, Shiwei Zhou†, Xiaodong Huang†, Dong Ruan‡, and Yi Min Xie† \\ ${ }^{\dagger}$ Centre for Innovative Structures and Materials, School of Civil, Environmental and \\ Chemical Engineering, RMIT University, \\ 394 Swanston Street, Melbourne CBD, Victoria, Australia, VIC3000 \\ ${ }^{\ddagger}$ Faculty of Engineering and Industrial Sciences, Swinburne University of Technology, \\ John Street, Hawthorn, VIC 3122, Australia
}

[Received date; Accepted date] - to be inserted later

\begin{abstract}
Auxetic metamaterials have enhanced indentation and penetration resistance due to their high shear strength and modulus. Its auxetic performance under dynamic loading cases is an important property for shields and armour applications. In the present study, compressive tests at different impact velocities on buckling-induced auxetic metamaterials were conducted for two different initial geometries. A photographic technique was applied to measure the Poisson's ratio. When the dynamic data are compared with those of quasi-static experiments, it is found that the NPR for the buckling-induced metamaterial is sensitive to the rate of loading, while the NPR for the metamaterial with initial auxetic behaviour is insensitive to the loading rate. It is also found that the deformation pattern is similar to that in the quasi-static loading condition when the impact force measured by the test machine is dominated by the inertia force of the metamaterials.
\end{abstract}

\section{INTRODUCTION}

Cellular materials are commonly used as packaging and cladding materials to protect products and personnel during collision and impact events ${ }^{1}$. The cellular materials with Negative Poisson's Ratio (NPR) have remarkable resilience under dynamic impact loading compared to the conventional foams ${ }^{2}$. The mechanical characterization of those auxetic cellular materials at varying strain rates is essential for their efficient use in dynamic loading applications ${ }^{3}$.

The earliest work on synthetic NPR metamaterials was a foam with re-entrant cells that unfolded in all directions under unidirectional tension ${ }^{4}$, after that it was found that NPR was a common phenomenon in many crystals as in cubic metals ${ }^{5}$. Most of the earlier work focused on periodic 2D geometries with NPR ${ }^{6}$ and mechanisms were revealed as chiral in microstructures and rotation of rigid units. ${ }^{7}$ Later on, analytical studies were conducted on 3D auxetic cellular materials consisting of networks of beams, ${ }^{8}$ multi-pods ${ }^{9}$ and rigid units ${ }^{10}$. Due to the limitation of fabrication techniques, only very few designs of synthetic 3D auxetic materials have been developed ${ }^{11}$. In all of these microstructures, the auxetic behaviour exhibited only in the limit of small strains, and the design of 3D auxetic systems capable of retaining the unusual properties at large strains still remains a challenge ${ }^{11,12}$. This limited its application in packaging and protective structures and materials, in which the large deformation is essential and inevitable. Most recently, a metallic 3D architecture based on a bow-tie functional element has been fabricated which extends the strain range for NPR to $0.1 .{ }^{13}$ Babaee et al. ${ }^{12}$ put forward a new design of 3D NPR bucklicrystals using various buckliball as building cell and extend the strain range further to 0.3 . However, the topology of their building cell was relatively complicated and the manufacture approach included extra bonding interface which resulted in scattering of the mechanical properties of their NPR materials. ${ }^{11}$ Most recently, inspired by a planar metamaterial with NPR 
behaviour induced by elastic instability, ${ }^{14,} 15$ the authors discovered the similar buckling-induced NPR behaviour in metamaterials with very simple building cells ${ }^{16}$, and they have more potential to be used in the applications mentioned above. For conventional cellular materials, the deformation pattern will transform from unformed mode to localised deformation mode as indicated in the previous research results ${ }^{17}$. For an effective NPR behaviour, the deformation needs to be uniform for better performance. Thus the NPR may become less obvious or even disappear when the loading rate increases. However, there is a lack of scientific data on the dynamic NPR behaviour of metamaterials with buckling-induced auxeticity.

In this paper, dynamic NPR behaviour of simple auxetic metamaterials was tested to investigate performance of this newly designed auxetic metamaterials. Uniaxial dynamic compressive tests were conducted at strain rates of $10^{1}$ and $10^{2} \mathrm{~s}^{-1}$ by using a High Rate Instron machine to study the dynamic NPR behaviour of the simple auxetic metamaterial. Two different auxetic specimens with different initial geometries were tested. The experimental results were discussed together with quasi-static data from our previous research ${ }^{16}$.

\section{EXPERIMENTS}

\subsection{Specimens and its base material}

The detailed Information on the design of the microstructures of this auxetic metamaterial can be found in the previous paper by the authors ${ }^{18}$.The specimens of bulk material were fabricated using 3D printing (Objet Connex350) with a silicone-based rubber material (TangoPlus FLX930) and a supporting material as shown in Figure 1 (a). The material properties of the printed TangoPlus mate-rial were measured through standard compression tests of six printed cylinders with same printing configurations as for bulk metamaterials. The compression process stopped at a true strain of $\varepsilon=0.70$. The results indicated that the constitutive behaviour could be accurately represented by a linear elastic model. It should be noted that the printed TangoPlus material exhibited slightly ani-sotropic behaviour. The Young's modulus along the printing direction, $0.925 \pm 0.02 \mathrm{MPa}$, was slightly lower than its modulus in the lateral direction, $1.05 \pm 0.03 \mathrm{MPa}$. The Poisson's ratio was found to be 0.48 .

According to the standard of compressive tests and recent research ${ }^{16}$ on other cellular materials, the specimen size effect is negligible for foams when the dimension of a specimen is sufficiently larger than the cell size, i.e. 7 times of the cell size for metallic foams. To obtain reliable homogenized material properties ${ }^{19}$, the test specimens had eight building cells in each normal direction. The dimensions were determined by the accuracy of the 3D printer and the difficulty of removing the supporting material without any damage to the microstructures of the designed model. The sizes are height $\times$ width $\times$ depth $=100 \times 100 \times 100 \mathrm{~mm}$. The original printed model contained supporting material, which must be carefully removed by a drilling process followed by flushing using water. In spite of extreme care being taken during the removal of the supporting material, several thinnest links in the bulk material with SC cells were broken. An epoxy adhesive was used to repair these damages. Due to the cost of the specimen, only two configurations were considered, i.e., originally designed simple auxetic metamaterial and the metamaterial shaped by $8 \%$ of buckling mode.

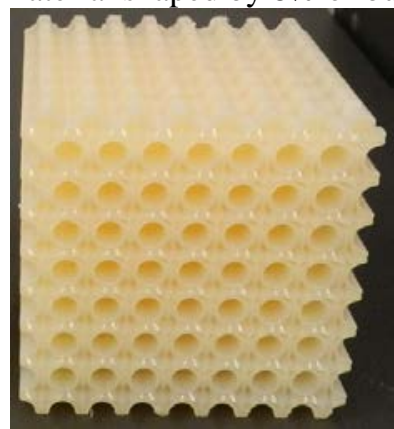

(a)

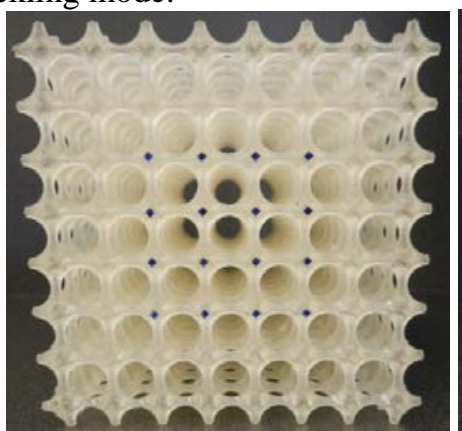

(b)

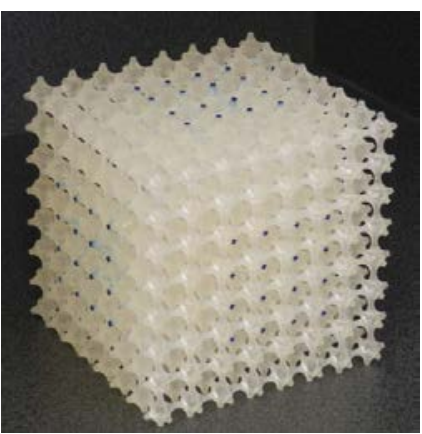

(c)

Figure 1. An example of the auxetic metamaterial specimens for impact tests (size: 100mmx100mmx100mm). (a). Printed model with supporting material; (b).Front view of final specimen;

(c). 3D view of final specimen 
Figure 1 shows a typical specimen for a dynamic compressive test. The highlighted points in the centre region were used to calculate the Poisson's Ratio. According to the quasi-static experiment, the deformation is purely elastic and reversible. Thus only two specimens were used to test its performance under different impact velocities. One specimen is originally designed auxetic metamaterials as shown in Figure 1. The other is a similar auxetic metamaterials with altered geometry by introducing $8 \%$ imperfection from the desired buckling mode.

\subsection{Experimental set-up}

The dynamic tests were performed using a High Rate Instron Test System. The dynamic uniaxial compressive tests were conducted at different strain rates, namely, 10 to $100 \mathrm{~s}^{-1}$ to obtain effect of loading rates on auxetic metamaterial under dynamic loading with different strain rates. A high rate method with different drive profiles was adopted. The drive profiles were created to control the end strain of the dynamic compression tests to protect the specimens from total failure. A photograph of the dynamic experimental setup is shown in Figure 2. The specimen was placed on the bottom compression platen which was positioned at a considerable long distance from the top platen at the beginning of each test. The specimen was accelerated to the required velocity across that distance before the top surface of the specimen touched the top platen. Double sided sticky tape was used to retain the specimen on the bottom platen and avoid any possible slippage of the specimen. The surface friction effect is an important factor for most compression test. To minimize this effect, the double sided glue was only applied to the centre region of the crosssection.

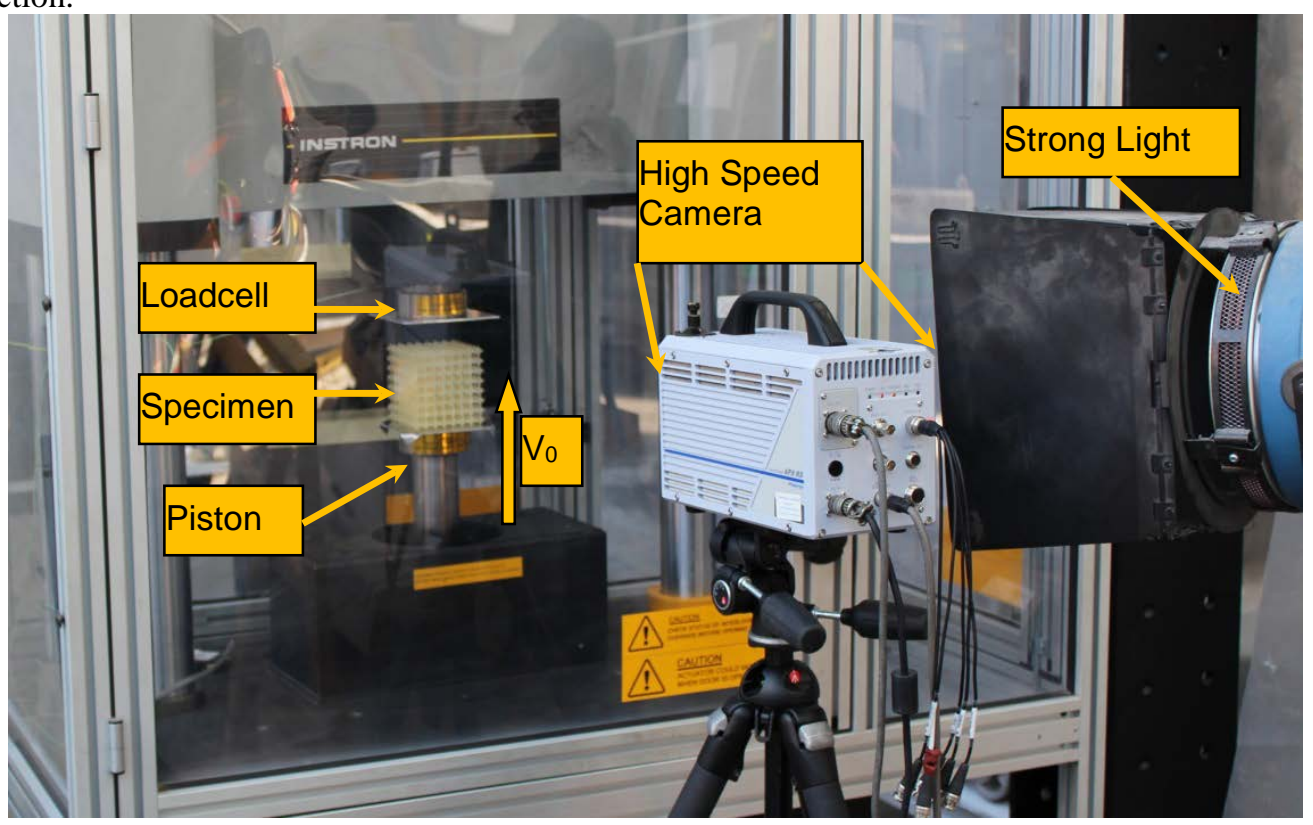

Figure 2. Experimental setup

For all the tests, a sampling rate of $500 \mathrm{kHz}$ was used for dynamic compression tests. The load history was measured by a Kistler load cell 9071A without data filter. The displacement history was measured by an inherent LVDT with data filter by setting cut off frequency of $1000 \mathrm{~Hz}$.

When the top surface of the specimen touched the top platen, the bottom platen and specimen began to decelerate, which resulted in a dropping strain rate during the compression process as shown in Figure3. High Rate Instron Test System provides a feedback mechanism by its FastTrack $^{\mathrm{TM}}$ VHS8800 controller to change the drive profile of the hydraulic system according to the force history from experimental data. Thus a relatively constant loading rate can be achieved during later experiments. The initial drive profile is a constant value. It can be increased according to the force history obtained by the load cell to obtain the new drive profile. The consistency of the strain rate during compression of the specimen can be improved further by iterating the procedure several times. However, due to the weak resistance of the TangoPlus specimen, compressive force of is too sensitive to the loading rate. Thus drive profiles obtained from specimens after two 
iterations were selected for all tests for each strain rate. The effect of drive profiles after iteration on force displacement curves was similar to that for aluminium foams in our previous study ${ }^{20}$.

High speed camera was used to capture the deformation process. The NPR was calculated from the centre region as shown in Figure 1(a) from those photographs.
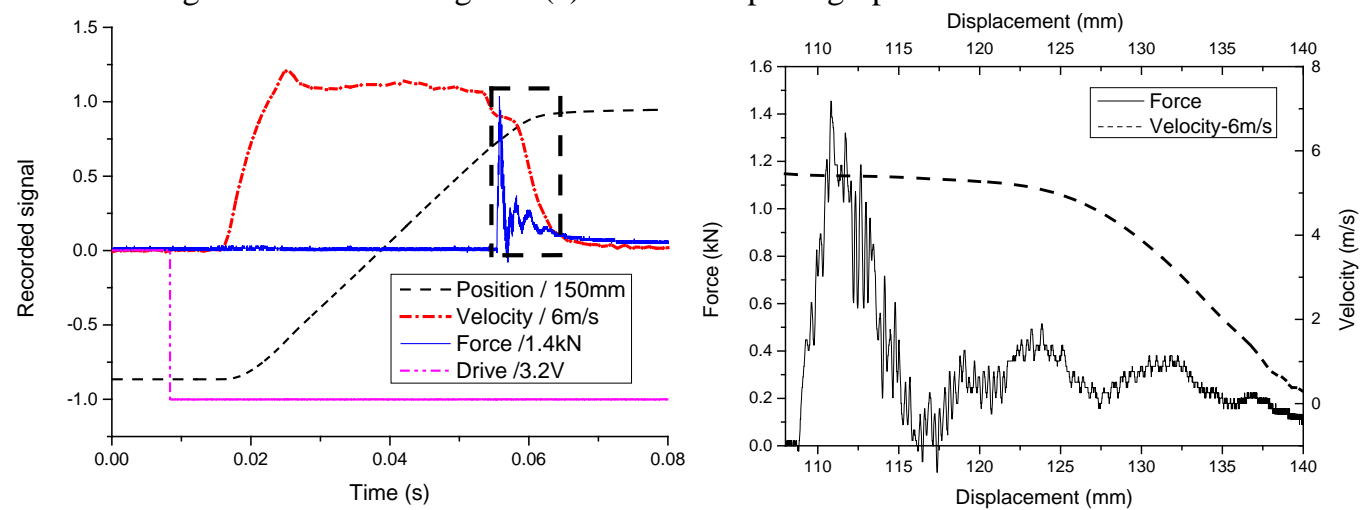

Figure 3. Overall impact processes and the velocity variation with respect to displacement of specimen. (The chart in the right hand is an enlarged view of the curves in the square with dash-line in the left chart).

\section{EXPERIMENTAL RESULTS}

\subsection{Deformation features at different loading rates}

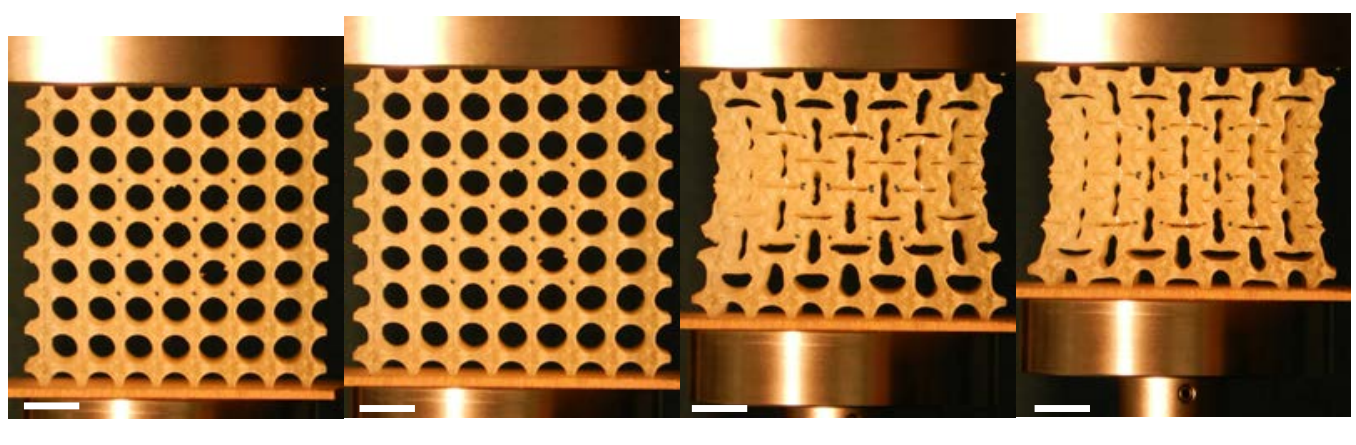

$\delta=0.0 \mathrm{~mm}$

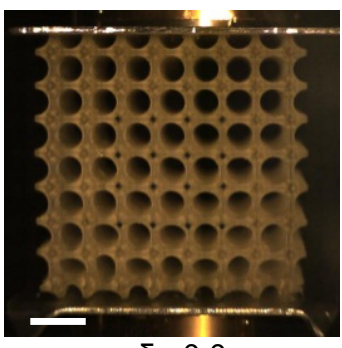

$\delta=0.0 \mathrm{~mm}$ $\delta=3 \mathrm{~mm}$

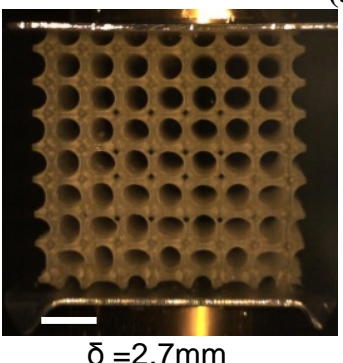

(a)

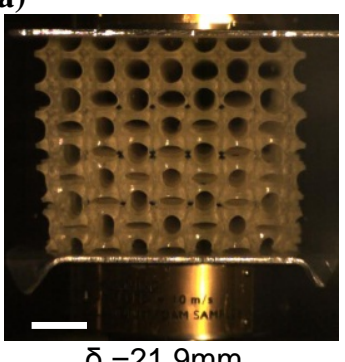

$\delta=21.9 \mathrm{~mm}$ $\delta=30 \mathrm{~mm}$

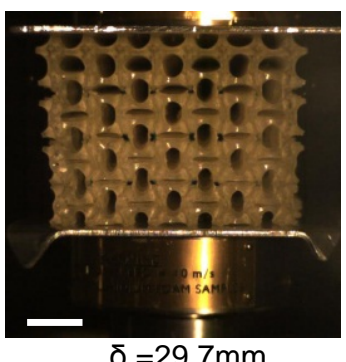

$\delta=29.7 \mathrm{~mm}$

(b)

Figure 4. Comparison of deformation patterns for buckling-induced auxetic metamaterial originally designed (scale bar: $20 \mathrm{~mm}$ ) (a). At quasi-static strain rate of $10^{-3} \mathrm{~s}^{-1}$; (b). At dynamic strain rate of $10^{2} \mathrm{~s}^{-1}$

For the buckling-induced auxetic metamaterials, the overall deformation was not uniform and was different from the deformation pattern observed at quasi-static loading rate ${ }^{18}$ as shown in Figure 4(a). At quasi-static strain rate, the axial deformation occurred first before buckling occurred at about a strain of 0.03 . Then, the microstructures buckled into a localised deformation pattern which can be described as ellipses with same sizes with its long axis being placed horizontally and vertically alternatively along both directions in a planar view as shown in Figure 
4. The magnitude of the localised deformation is larger in the centre region than that in the contact regions with top and bottom platen. While for the deformation pattern under dynamic strain rate, the deformation started from the surface to the bottom platen even before the specimen contacted with the top platen, which indicated that the inertia force during the accelerating process will cause obvious deformation in the specimen. Thus the inertia force is not negligible. The deformation propagated from the bottom to the top of the specimen gradually. No obvious crush band was observed for tests at impact velocities ranging from $1 \mathrm{~m} / \mathrm{s}$ to $10 \mathrm{~m} / \mathrm{s}$. The alternating ellipses deformation mode occurred at the quasi-static loading rate ${ }^{18}$ occurred gradually with the propagation of the deformation. At the same time the ellipses with vertical long axes deformed slower than that with horizontal long axes. This trend became more obvious when the impact velocity increased as shown in front view of the specimen at an initial impact velocity of $10 \mathrm{~m} / \mathrm{s}$ in Figure 4. It indicated that the absolute value of NPR became smaller with the increasing impact velocity.

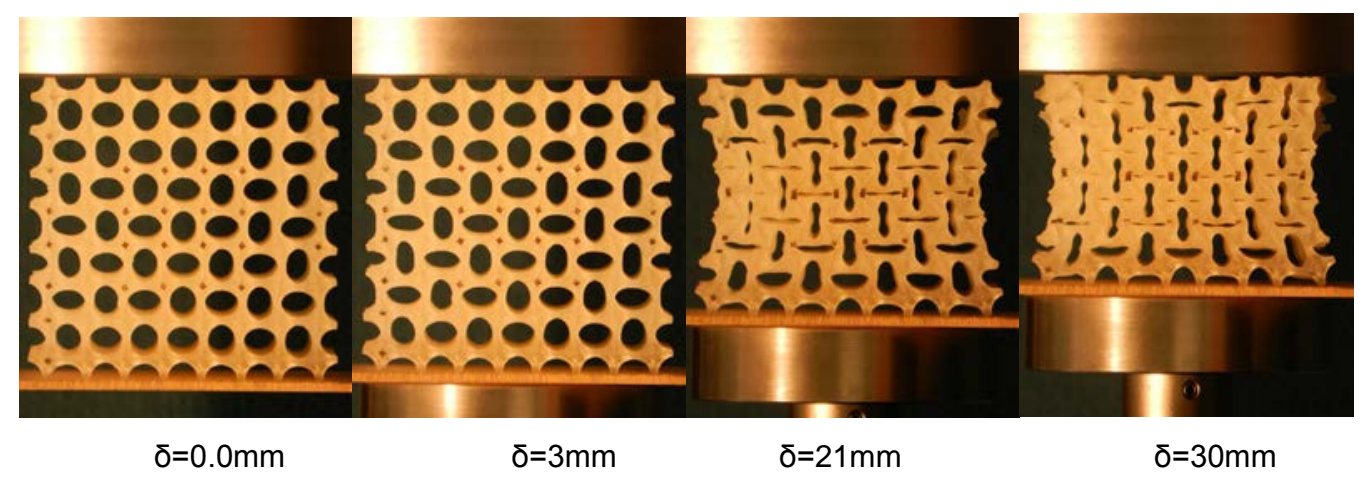

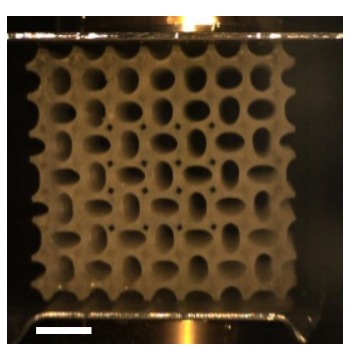

$\delta=0.0 \mathrm{~mm}$

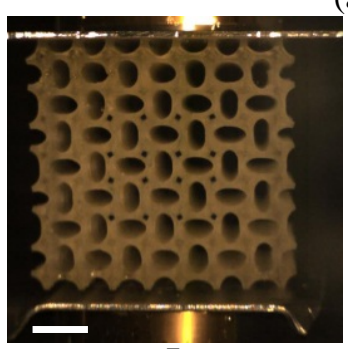

$\delta=3 \mathrm{~mm}$ (a)

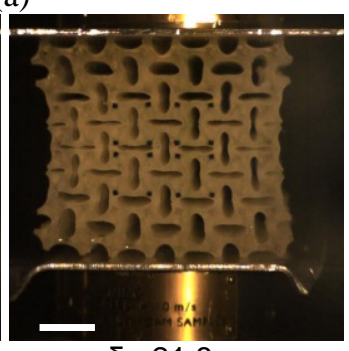

$\delta=21.8 \mathrm{~mm}$

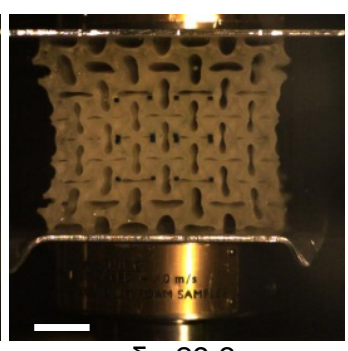

$\delta=29.3 \mathrm{~mm}$

(b)

Figure 5: Comparison of deformation patterns for altered auxetic metamaterial (scale bar: $20 \mathrm{~mm}$ ) (a). At quasi-static strain rate of $10^{-3} \mathrm{~s}^{-1} ;(\mathrm{b})$. At dynamic strain rate of $10^{2} \mathrm{~s}^{-1}$

For the auxetic metamaterials with altered geometry by $8 \%$ imperfection from the buckling mode, the overall deformation is more uniform than the previous specimen for all impact velocities as shown in Figure 5. The deformation started from the centre of the specimen and then propagated to the top and bottom parts, which was similar to the deformation patterns observed at quasi-static strain rate of $10^{-3} \mathrm{~s}^{-1}$ in Figure 5(a). The deformation was nearly uniform in the centre region of the specimen for all impact velocities. Due to the influence of the friction force on the top and bottom surfaces, the construction cells deformed less at region closet to top and bottom platens. Contrast to buckling-induced metamaterial, the altered buckling-induced metamaterial have enhanced NPR behaviour under dynamic loading. The alternating ellipses deformation appeared to deform simultaneously rather than sequentially. The ellipses with vertical long axes deformed in the similar magnitude as that with horizontal long axes. No localised crush bands were observed at all impact velocities.

\subsection{Variation of NPR with respect to stain}

The Poisson's Ratio (PR) was calculated from four points in the centre region of the tested specimen in one surface. For each point such as P1 in Figure 6, the original horizontal length of this point was taken as $\mathrm{X} 2-\mathrm{X} 1$ and the original vertical length as $\mathrm{Y} 2-\mathrm{Y} 1$ where $\mathrm{Xi}$ and $\mathrm{Yi}$ is the coordinate of the adjacent points around P1. The PR was calculated as the negative ratio of the length change in horizontal direction and vertical direction. 


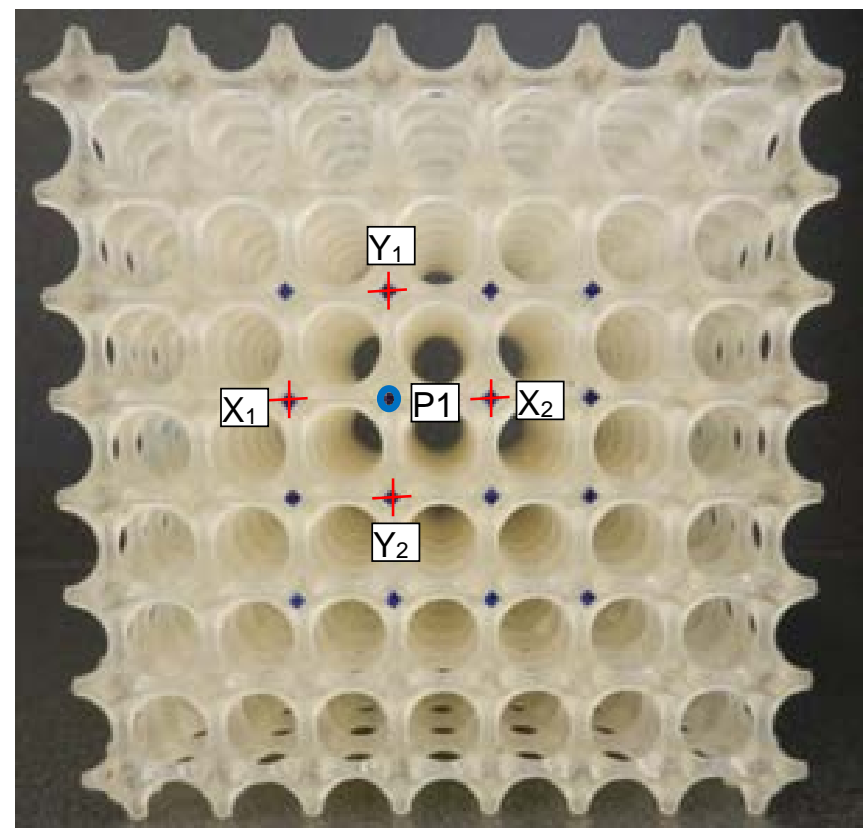

Figure 6. Illustration of calculating PR in the centre region
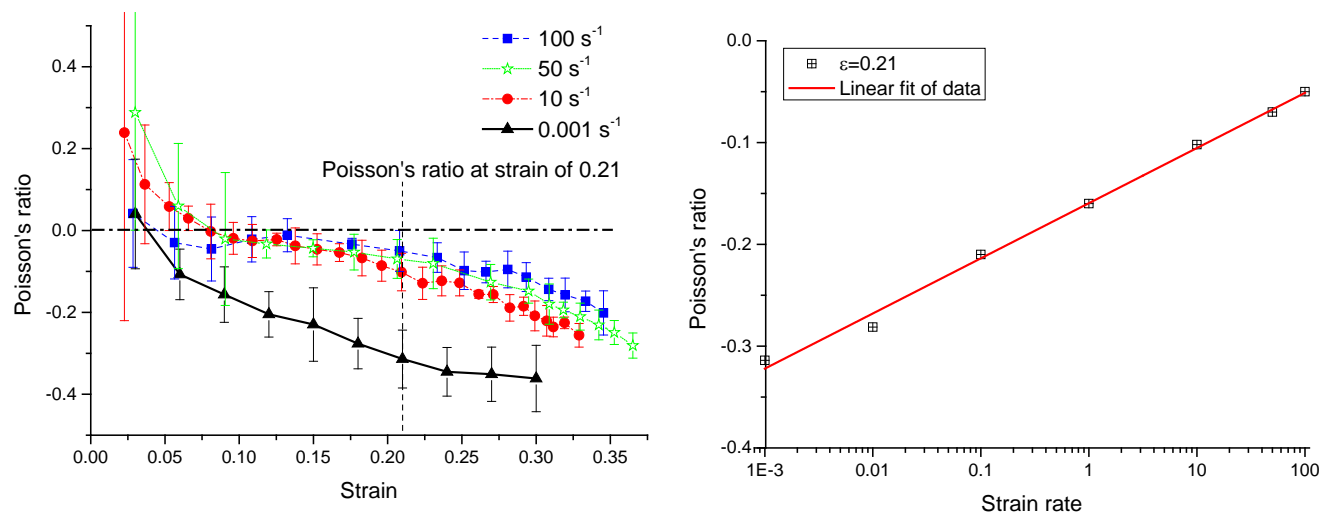

Figure 7. Variation of NPR with respect to strain at different strain rate for buckling-induced auxetic metamaterial

Figure 7(a) shows the variation of the NPR with respect to strain for buckling-induced metamaterial at different strain rate. Similar to that at quasi-static loading, the PR is positive during the compression test before buckling occurred. Only after buckling occurred, auxetic behaviour became evident. The PR exhibited a nonlinear relationship with respect to the evolution of strain with obvious decreasing trend. For most impact velocities, the buckling occurred later due to the lateral inertia effect of the microstructures. It should be noted that this phenomenon was diminished by the deformation of specimen due to the inertia force during the acceleration process of the piston before the impact event. The auxetic behaviour became less obvious with decreasing absolute value of NPR at high loading rate as shown in Figure 7(b). Thus for buckling-induced NPR materials, increasing the loading rate has significant negative effect on the NPR behaviour. It may cause the loss of NPR behaviour if the impact velocity increases further. When the value of NPR was plotted against the strain rate, a roughly linear relationship was found when the strain rate is in logarithmic coordinate as shown in Figure 7 (b). 

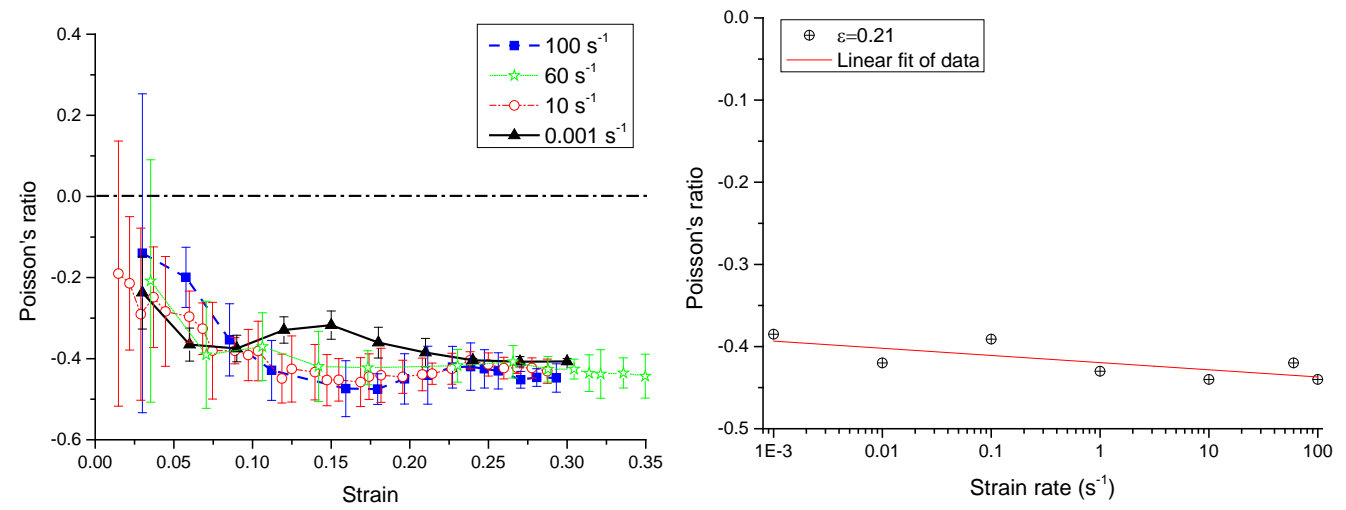

Figure 8. Variation of NPR with respect to strain at different impact velocities for altered NPR metamaterial

For the auxetic metamaterials with altered geometry by $8 \%$ imperfection of the desired buckling mode, the NPR behaviour was observed from the beginning of the compression tests at all impact velocities. The overall PR and strain curve exhibited a nonlinear decreasing trend. The value of NPR are similar at different strain rates as shown in Figure 8 (b). This trend indicated that the effect of strain rate on the NPR behaviour for this type of metamaterials is negligible.

\subsection{The effect of inertia force on the compressive force and NPR behaviour of auxetic metamaterials}

Force and displacement histories were obtained from the Instron machine (VHS 8800). The nominal stress strain curves are shown in Figure 9. The apparent dynamic stress strain curves were totally different from these at quasi-static loading rate. Different from other cellular materials with a steep elastic region followed by a plateau collapse region up to densification, the nominal stress strain curve exhibited an initial peak force followed by a plateau force with large fluctuation and pretty low stress level compared with the initial peak stress. No obvious densification was observed even when the strain was up to $40 \%$. The initial peak stress, plateau stress and fluctuation were all increasing with the increasing loading rate. For buckling-induced metamaterial in Figure 9, l the peak stress at quasi-static loading was 0.0013MPa, which was 55 times less than the peak stress $(0.071 \mathrm{MPa})$ when the impact velocity was $1 \mathrm{~m} / \mathrm{s}$ and it was 279 times less than the peak stress $(0.363 \mathrm{MPa})$ when the impact velocity was $10 \mathrm{~m} / \mathrm{s}$. For altered metamaterial with $8 \%$ imperfection in Figure 10, 1 the peak stress at quasi-static loading was $0.00076 \mathrm{MPa}$, which was 34 times less than the peak stress $(0.026 \mathrm{MPa})$ when the impact velocity was $1 \mathrm{~m} / \mathrm{s}$ and it was 376 times less than the peak stress $(0.290 \mathrm{MPa})$ when the impact velocity was $10 \mathrm{~m} / \mathrm{s}$.

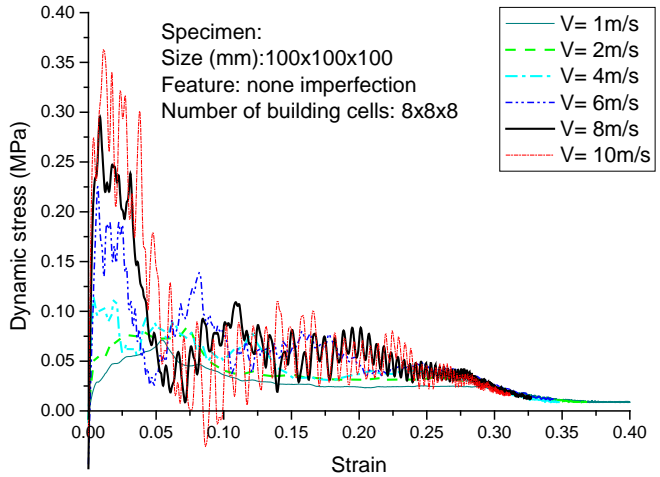

(a)

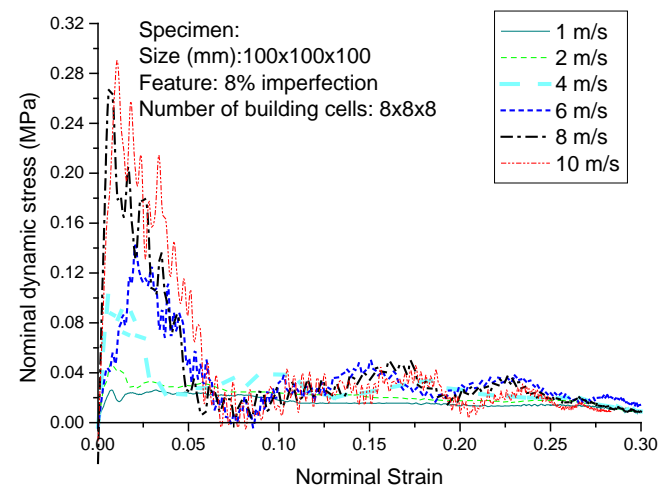

(b)

Figure 9. Nominal stress strain curves for metamaterials at different impact velocity. (a) Buckling-induced auxetic metamaterial. (b) Altered auxetic metamaterial with 8\% of imperfection of desired buckling mode. 
Comparison between the nominal dynamic strain-stress curves, the stress was higher for the buckling-induced auxetic metamaterials. As shown in Figure 2, the load cell was fixed on the top platen. Therefore, the measured force included the inertial force (the force required to stop the specimen from moving at given initial velocity) of the specimen. The average stress enhancement in the plateau collapse region caused by the inertia of specimen can be estimated using the theorem of momentum as

$$
F t=m v \Rightarrow F=\frac{m}{t} v=\frac{\rho_{0} A L}{\frac{L \varepsilon_{d}}{v}} v=A \frac{\rho_{0} v^{2}}{\varepsilon_{d}} \text { and } \quad \sigma_{i} A=F
$$

thus,

$$
\sigma_{i}=\frac{\rho_{0} v^{2}}{\varepsilon_{d}}
$$

where $\sigma_{i}$ is the nominal stress enhancement caused by the inertia effect, $A$ is the cross sectional area, $L$ is the initial length of the specimen, $\rho_{0}$ is the density of , $v$ is the compression velocity, $t$ is the duration of the inertial force, and $\varepsilon_{d}$ is the densification strain. The expression is identical to that obtained from 1D structural shock model which was put forward by Reid et al. ${ }^{21}$ and further developed by Harrigan et al. for cellular materials ${ }^{22}$. It should be noted that the deformation pattern of the auxetic metamaterials did not satisfy the requirements for 1D structural shock model in the highest loading velocities, i.e. $10 \mathrm{~m} / \mathrm{s}$. For example, there is no obvious shock front and the crush process of the metamaterials is not sequential from the impact end etc. However, this estimation gives an average enhancement for the effect of inertial force on the plateau stress of cellular material under compression.

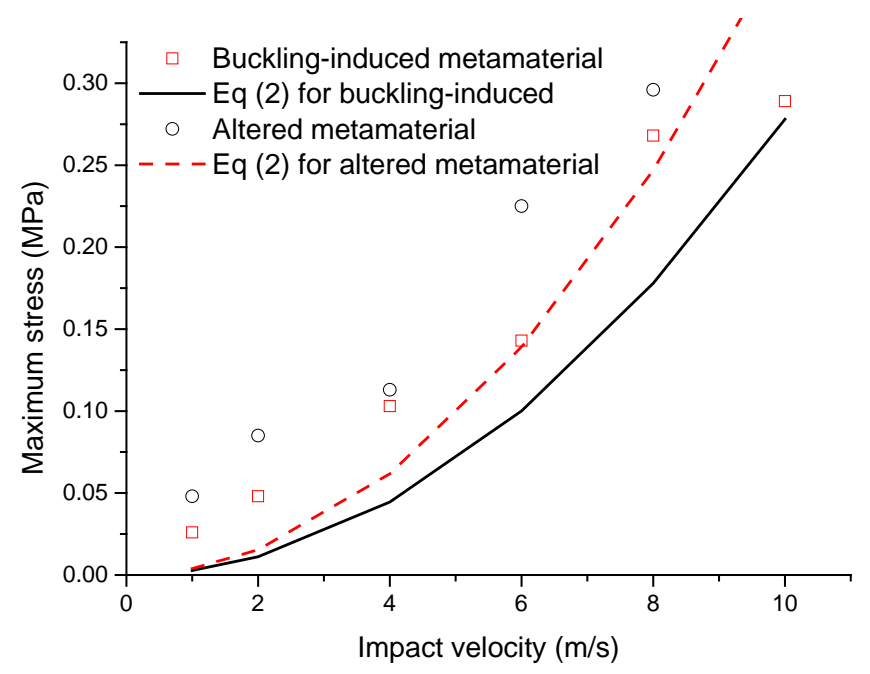

Figure 10. Estimation of the inertia effect on the auxetic metamaterials

Consider two extreme cases with maximum inertial force for our tests, i.e. $\rho_{0}=139 \mathrm{~kg} / \mathrm{m}^{3}$,

$\varepsilon_{d}=0.3, V_{0}=10 \mathrm{~m} \cdot \mathrm{s}^{-1}$ the value of stress $\sigma_{i}$ is $0.046 \mathrm{MPa}$, which is much more larger than the measured static plateau stress, $0.0011 \mathrm{MPa}$. It can be seen the peak force occurred only in a limited strain range, i.e. 0.05 . If we change $\varepsilon_{d}=0.05$, the corresponding value is $0.23 \mathrm{MPa}$ which is close to the peak stress as shown in Figure 10. Thus the dynamic stress enhancement observed our experiments was mainly caused by the inertia force. It should be noted that the density for the altered metamaterials, i.e., $193 \mathrm{~kg} / \mathrm{m}^{3}$, was larger than that for the buckling-induced metamaterials. However, the peak force for the altered metamaterial was less than that of the buckling-induced metamaterials. Thus the deformation patter should also contribute to the stress enhancement. According to previous studies on cellular materials, when the inertia force was the 
dominate force for the deformation process, the deformation pattern will be totally different from that at the quasi-static loading rate. Usually it changed from uniformed deformation pattern to localised deformation pattern. It did not occur in our experiments. The mechanisms behind this phenomenon will be clarified using FEA in our future work.

\section{CONCLUSIONS}

The auxetic behaviour of two types of auxetic metamaterials was investigated in this paper by conducting experiments at different impact velocities on cubic specimens of two newly designed auxetic metamaterials. The variation of the NPR with respect to stain curves at different loading rates was obtained at the macroscopic level. The following conclusions can be drawn from our investigations.

(1) For buckling-induced auxetic metamaterial, the auxetic behaviour indicated by the absolute value of NPR became less obvious under high velocity impact;

(2) For altered auxetic metamaterial, the strain rate has negligible effect on the auxetic behavior;

(3) The dynamic enhancement for peak stress was very prominent, which is 2 orders larger than the peak force at quasi-static loading rate. The underlying mechanism can be explained by inertia force from the specimen in the current experimental configuration;

(4) The inertia dominated deformation process under high velocity impact did not change the deformation pattern for altered auxetic metamaterials from that at quasi-static loading rate;

(5) The stable performance of altered auxetic metamaterials at different strain rates are in favour of their application in various engineering fields such as packaging, acoustic and vibration isolation, and impact energy absorption against localised indentation and penetration.

\section{ACKNOWLEDGEMENTS}

This work was supported by the Australian Research Council (DE120102906, DP140100213), the National Natural Science Foundation of China (51228801) and the Open Research Fund Program of the State Key Laboratory of Advanced Design and Manufacturing for Vehicle Body (41215001).

\section{REFERENCES}

1. Gibson L. J., Ashby M. F. \& Harley B. A. Cellular materials in nature and medicine, Cambridge University Press, Cambridge, 2010.

2. Prawoto Y. Seeing auxetic materials from the mechanics point of view: A structural review on the negative Poisson's ratio, Computational Materials Science, 2012, 58, 140153.

3. Critchley R., et al. A review of the manufacture, mechanical properties and potential applications of auxetic foams, Physica Status Solidi B, 250, 2013, 1963-1982.

4. Lakes R. Foam structures with a negative Poisson's Ratio, Science, 1987, 235, 10381040 .

5. Baughman R., Shacklette J., Zakhidov A. \& Stafstrom S. Negative Poisson's ratios as a common feature of cubic metals, Nature, 1998, 392, 362-365.

6. Liu Y. \& Hu H. A review on auxetic structures and polymeric materials, Scientific Research Essays, 2010, 5, 1052-1063.

7. Overvelde J. T. B., Shan S. \& Bertoldi K. Compaction Through Buckling in 2D Periodic, Soft and Porous Structures: Effect of Pore Shape, Advanced Materials, 2012, 24, 2337 2342.

8. Hughes T. P., Marmier A. \& Evans K. E. Auxetic frameworks inspired by cubic crystals, 2010, International Journal of Solids and Structures, 2010, 47, 1469-1476.

9. $\quad$ Pikhitsa P. V., Choi M., Kim H. J. \& Ahn S. H. Auxetic lattice of multipods, Physica Status Solidi B, 2009, 246, 2098-2101.

10. Bückmann T., et al. Tailored 3D Mechanical Metamaterials Made by Dip-in Direct- 
Laser-Writing Optical Lithography, Advanced Materials, 2012, 24, 2710-2714.

11. Lee J.-H., Singer J. P. \& Thomas E. L. Micro-/Nanostructured Mechanical Metamaterials, Advanced Materials, 2012, 24, 4782-4810.

12. Babaee S., et al. 3D soft metamaterials with negative Poisson's ratio, Advanced Materials, 2013, 25, 5044-5053.

13. Bückmann T., et al. Tailored 3D Mechanical Metamaterials Made by Dip-in DirectLaser-Writing Optical Lithography, Advanced Materials, 2012, 24, 2710-2714.

14. Bertoldi K., Boyce M. C., Deschanel S., Prange S. M. \& Mullin T. Mechanics of deformation-triggered pattern transformations and superelastic behavior in periodic elastomeric structures, Journal of the Mechanics and Physics of Solids, 2008, 56, 26422668.

15. Willshaw S. \& Mullin T. Pattern switching in two and three-dimensional soft solids, Soft Matter, 2012, 8, 1747-1750.

16. Idris M. I., Vodenitcharova T. \& Hoffman M. Mechanical behaviour and energy absorption of closed-cell aluminium foam panels in uniaxial compression, Materials Science and Engineering: A, 2009, 517, 37-45.

17. Ruan D., Lu G., Wang B. \& Yu T. X. In-plane dynamic crushing of honeycombs-a finite element study. International Journal of Impact Engineering, 2003, 28, 161-182.

18. Shen J., Zhou S., Huang X. \& Xie Y. M. Simple cubic three-dimensional auxetic metamaterials. Physica Status Solidi (b) , 2014, 251(8), 1515-1522.

19. Ashby M. F., et al. Metal Foams: A Design Guide, Butterworth-Heinemann, Warrendale, 2000.

20. Shen J., Lu G. \& Ruan D. Compressive behaviour of closed-cell aluminium foams at high strain rates. Composites Part B Engineering, 2010, 41, 678-685.

21. Reid S. R., Bell W. W. \& Barr R. A. Structural plastic shock model for one dimensional ring system. International Journal of Impact Engineering, 1983, 1, 175-191.

22. Harrigan J. J., Reid S. R., Tan P. J. \& Reddy T. Y. High rate crushing of wood along the grain. International Journal of Mechanical Sciences, 2005, 47, 521-544. 